\title{
Corela
}

Cognition, représentation, langage

HS-27 | 2019

Co(n)textualisation(s)

\section{Contextualisations d'extraits de jeux vidéo et prises en compte des ressources multimodales pour une analyse des pratiques interactionnelles entre joueurs}

Isabel Colón de Carvajal

\section{CpenEdition}

Journals

Édition électronique

URL : http://journals.openedition.org/corela/7682

DOI : $10.4000 /$ corela.7682

ISSN : 1638-573X

Éditeur

Cercle linguistique du Centre et de l'Ouest - CerLICO

Référence électronique

Isabel Colón de Carvajal, « Contextualisations d'extraits de jeux vidéo et prises en compte des ressources multimodales pour une analyse des pratiques interactionnelles entre joueurs », Corela [En ligne], HS-27 | 2019, mis en ligne le 30 mars 2019, consulté le 01 mai 2019. URL : http:// journals.openedition.org/corela/7682 ; DOI : 10.4000/corela.7682

Ce document a été généré automatiquement le 1 mai 2019.

\section{(i) (ㅇ)

Corela - cognition, représentation, langage est mis à disposition selon les termes de la licence Creative Commons Attribution - Pas d'Utilisation Commerciale - Partage dans les Mêmes Conditions 4.0 International. 


\title{
Contextualisations d'extraits de jeux vidéo et prises en compte des ressources multimodales pour une analyse des pratiques interactionnelles entre joueurs
}

\author{
Isabel Colón de Carvajal
}

\section{Remerciement}

L'auteure remercie le LABEX ASLAN (ANR-10-LABX-0081) de l'Université de Lyon pour son soutien financier dans le cadre du programme «Investissements d'Avenir » (ANR-11-IDEX-0007) de l'État Français géré par l'Agence Nationale de la Recherche (ANR).

\section{Introduction}

1 Notre travail s'inscrit dans les ancrages théoriques de l'ethnométhodologie et de l'analyse conversationnelle. Notre objet de recherche est d'analyser les pratiques interactionnelles entre joueurs en situation de jeux vidéo (Colón de Carvajal, 2013, 2016; Mondada, 2011, 2012 ; Piirainen-Marsh \& Tainio, 2009a et b) ; et de comparer différents jeux sur le plan de leur organisation interactionnelle (les cadres de participation) ainsi que leur structure séquentielle et multimodale (l'organisation de l'espace et la gestion des objets).

2 Ce qui peut sembler simple dans la pratique des joueurs se révèle très complexe au niveau de l'organisation de l'interaction. Premièrement, les joueurs interagissent principalement à travers un objet (numérique ou non) et jouent selon des règles définies en amont, ce qui nous oblige à questionner des concepts clé de l'analyse interactionnelle tels que le turn taking ou la séquence. Deuxièmement, dans certains jeux, les joueurs incarnent des personnages (avatars) qui réalisent des actions dans le jeu ce qui nous amène à nous interroger sur la relation entre joueur et avatar (Baldauf-Quilliatre \& Colón de Carvajal, 2015). Troisièmement, les joueurs agissent dans différents cadres de participation et 
passent d'un cadre à l'autre. Ils occupent donc différentes positions à différents moments $\mathrm{du}$ jeu (joueur et spectateur, mais aussi joueur engagé totalement dans le jeu, membre d'une équipe, co-participant dans l'interaction des joueurs, etc.). Les pratiques interactionnelles permettant de passer d'un rôle à l'autre (Baldauf-Quilliatre \& Colón de Carvajal, accepté, en préparation) méritent d'être analysées en détail.

3 Ainsi, à partir de ces pratiques de joueurs, nous souhaitons questionner dans cet article les problèmes et contraintes rencontrés, d'une part lors des analyses de ces données en situation de jeux vidéo, et d'autre part lors de présentations académiques de nos recherches. Une étude en cours, sur les séquences d'encouragement et d'instruction dans les interactions entre joueurs, nous permet de montrer et d'expliquer qu'il est indispensable de contextualiser le type de jeu joué, ses règles, ses options parfois spécifiques, les relations entre les joueurs, les rôles de chacun à l'instant de l'extrait, et aussi les actions en cours dans l'univers du jeu lui-même (un objet qui tombe, un avatar touché, etc.) qui donneront du sens à l'analyse pour le public, pour le lecteur.

Par exemple, si nous analysons l'encouragement «allez/ vas-y benoit \" hors de son contexte interactionnel immédiat, nous pourrions dire minimalement qu'il est syntaxiquement construit par une interjection suivie d'une forme impérative associée au prénom du joueur adressé. Cependant, d'un point de vue interactionnel et séquentiel, il est nécessaire d'apporter au lecteur des éclairages sur les tours précédant cet encouragement pour expliquer sa production à cet instant du jeu. Pour guider le lecteur de l'analyse, nous devons donc contextualiser par les informations suivantes: Benoit a choisi un gorille comme avatar. Il est en train de perdre (score en sa défaveur sur l'écran $\mathrm{du}$ jeu). Il rejette la faute sur son avatar. Un encouragement est alors explicitement adressé à Benoit.

Ces contextualisations et prises en compte des ressources multimodales, que le chercheur doit savoir doser, permettent d'expliquer et de décrire plus clairement et objectivement le phénomène linguistique, l'action ou encore la problématique analysée.

6 Après avoir présenté le corpus et le projet dans lequel s'inscrit cette recherche, nous analyserons deux extraits d'interaction entre joueurs permettant d'expliciter les indices de contextualisation indispensables à présenter au public. Notre étude souhaite contribuer à mieux comprendre les manières dont tout ce travail de contextualisation transforme à la fois le travail de recherche, le travail de recueil du corpus, mais aussi le traitement analytique des données et la présentation des extraits lors de communication pendant des conférences (en appui au clip vidéo joué) ou lors de la rédaction d'article pour des publications scientifiques (sans accès au clip vidéo joué).

\section{Données et approches méthodologiques}

7 Les données utilisées dans notre article s'inscrivent dans un projet de recherche mené par des membres de l'équipe InSitu: "INteractions : SItuations, praTiques et oUtils" du laboratoire ICAR ${ }^{1}$. Ce projet, financé par le programme ANR “jeunes chercheurs" jusqu'en 2014, s'intitulait «Ludespace : les espaces du jeu vidéo en France »². Il avait pour objectif de proposer un panorama et une cartographie des pratiques des jeux vidéo en France, dans une réflexion pluridisciplinaire menée entre des chercheurs issus de différents domaines en sciences humaines et sociales. 
Une première partie des enregistrements a été réalisée entre le printemps et l'automne 2012. Nous avons au minimum deux vues d'une même situation de jeux vidéo : i) le joueur dans son espace; ii) l'écran du joueur. Pour certaines situations, nous avons la vue supplémentaire des mains du joueur manipulant le contrôleur de la console (ou le clavier et la souris de l'ordinateur), et une source d'enregistrement audio. Au total, nous disposons de $18 \mathrm{~h}$ de données (vue joueurs, vue écran) de jeux vidéo (sur console). Dans cet article, nous analyserons deux situations de jeu: l'une sur Wii avec quatre joueurs dans la même équipe jouant face à un écran commun; et l'autre sur Playstation : avec deux joueurs en compétition jouant face à un écran commun divisé en deux zones (une pour chaque joueur).

Dans le projet Ludespace, nous avons adopté la méthodologie de travail de terrain et de recueil de corpus développée en linguistique interactionnelle et analyse conversationnelle qui donne à voir des expériences vidéoludiques variables selon les contextes d'activité. Cette méthodologie vise à capturer des données audio et vidéo afin de rendre disponibles, et donc analysables, les détails linguistiques, multimodaux et situationnels (regards, gestes, mouvements, actions, objets, cadre physique) pertinents pour l'interaction enregistrée (Groupe ICOR 2006). Elle permet aussi d'observer précisément l'évolution dans le temps et dans l'espace d'une ou plusieurs sessions de jeu, les interactions sociales et spatiales qui se nouent entre les joueurs et le support de jeu, entre les joueurs et leur environnement, et enfin entre les joueurs eux-mêmes.

\section{Contextualiser les extraits présentés}

Lors d'une analyse interactionnelle d'une problématique particulière, il est d'usage d'inscrire nos propos en présentant l'ancrage théorique dans lequel se positionne le chercheur et de proposer une revue de littérature pertinente des notions clés sur lesquelles porte notre recherche. L'étude plus générale que nous menons sur les données jeux vidéo, et qui sous-tend la problématisation de la contextualisation proposée dans cet article, porte sur l'analyse linguistique d'énoncé d'encouragement ou d'instruction produit par des joueurs et/ou des spectateurs. Notre propos ne sera donc pas de présenter les travaux existants sur la notion d'encouragement ou d'instruction en interaction, mais bien de nous focaliser sur les contraintes et les difficultés que nous rencontrons lorsque nous devons introduire et présenter, dans une communication et/ou dans un article, le corpus et les extraits de jeu vidéo sur lesquels est construite notre analyse. Dans cette partie nous distinguerons deux types d'informations qui relèvent, de notre point de vue, de la contextualisation nécessaire des extraits. Nous avons, d'une part, ce que nous appellerons les «métadonnées » générales sur le jeu; et d'autre part, des descriptions plus précises de l'extrait choisi en lien avec la problématique étudiée.

\section{1. Métadonnées générales sur le jeu}

\section{1. 1. Extrait $1:$ « vite vite vite/ vite vite vite/»}

11 Le premier énoncé d'encouragement analysé est tiré d'une session de jeu vidéo sur Wii, où quatre joueurs sont en train de faire une partie de New Super Mario Bros (Nintendo, 2009). Pris hors de son contexte d'énonciation, cet énoncé peut être syntaxiquement présenté et expliqué au public-lecteur comme un énoncé construit par un adverbe répété 
six fois. Nous pourrions dire aussi que le joueur Lucas produit cet énoncé d'encouragement vers la fin de l'extrait sélectionné (cf. image 1).

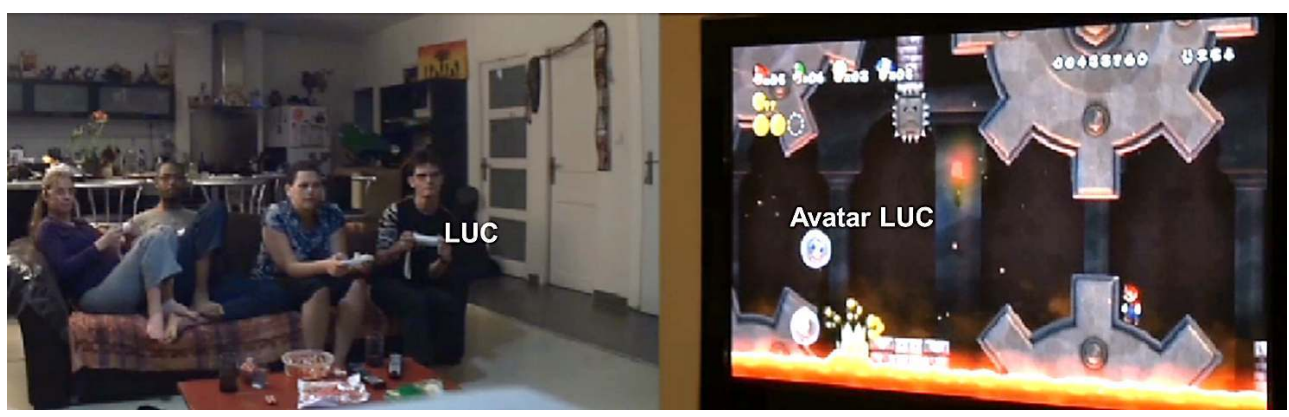

Image 1 : situation du jeu Mario - Lucas produit un encouragement

Cependant, nous nous inscrivons dans le domaine de l'analyse conversationnelle et de la linguistique interactionnelle, et de ce point de vue-là, ce qui nous intéresse est le contexte de production de l'énoncé d'encouragement produit par Lucas. Quand nous parlons de contexte de production ici, nous évoquons notamment les événements ou actions qui ont précédé l'encouragement, la motivation qui a incité le locuteur (joueur ou spectateur) à produire cet encouragement, l'effet que l'encouragement peut avoir ou non dans la pratique du jeu en cours, pour le joueur qui le reçoit, ou pour l'équipe dans le cas de la session sur Mario dans cet extrait.

Pour introduire et expliquer ces éléments de contexte propre à l'extrait analysé, il est donc nécessaire, au préalable, de donner au public-lecteur des informations générales sur le jeu que nous appelons «métadonnées ». Ces informations concernent principalement les prénoms des joueurs, leur nombre dans la session et la configuration des équipes le cas échéant. Pour l'extrait 1 : la session de jeu de Mario est composée de quatre joueurs : Léa, Dominique, Véro et Lucas, qui jouent dans la même équipe. Aussi, nous ajoutons systématiquement, que ce soit en communication orale ou dans l'écriture d'un article, une capture d'écran des joueurs en regard des avatars qu'ils contrôlent dans le jeu. Cette ressource iconographique (cf. image 2) permet au public-lecteur d'identifier par euxmêmes les avatars qui évoluent dans le jeu, associés au prénom des joueurs que nous mobilisons régulièrement par la suite dans l'analyse de l'extrait.

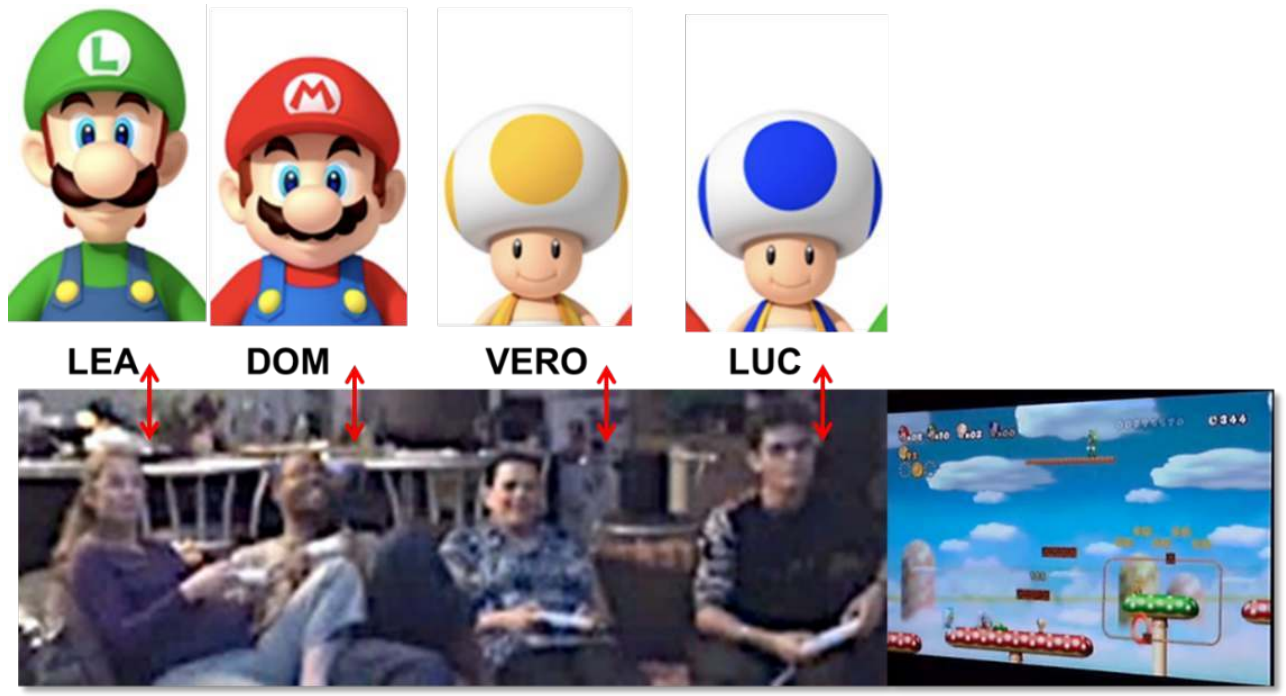

Image 2 : les quatre joueurs de Mario sur Wii, associés à leurs avatars 
Dans un second temps, nous expliquons l'objectif du jeu à ce moment précis de l'extrait. Dans l'extrait 1, les joueurs doivent passer des blocs de pierre piquants qui tombent du plafond tout en évitant la lave et en sautant sur des roues. Pour aider à la compréhension de l'univers du jeu, nous illustrons cette information par une représentation du « bloc de pierre piquant $»$ (cf. image 3 ).

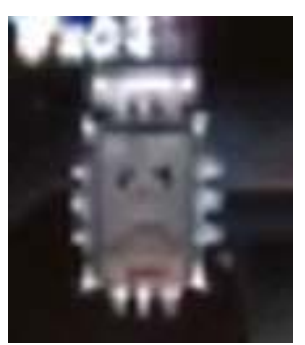

Image 3 : bloc de pierre piquant

\section{1. 2. Extrait $2:$ « allez :/ vas-Y benoit » ; « fais carré ENfoncé/ fais carré ENfoncé »}

Le deuxième extrait analysé dans l'article est tiré d'une session de jeu vidéo sur Playstation, où deux joueurs se battent dans le jeu Dragon Ball Z Budokai Tenkashi 3 (Atari, 2007), et trois spectateurs sont présents et peuvent commenter le jeu. Cet extrait comprend deux énoncés qui nous intéressent du point de vue de notre recherche sur les encouragements et instructions dans les jeux vidéo: il y a d'abord l'énoncé d'encouragement "allez/ vas-Y benoit» suivi de l'instruction "fais carré ENfoncé ». Syntaxiquement analysé, l'encouragement est construit par une forme impérative associée au prénom du joueur adressé; alors que l'instruction est composée d'un verbe directif qui porte sur une action technique du dispositif (sur le contrôleur). Aussi, nous pourrions préciser que Rodrigue produit l'encouragement adressé à Benoit, suivi par l'instruction de Xavier (cf. image 4).

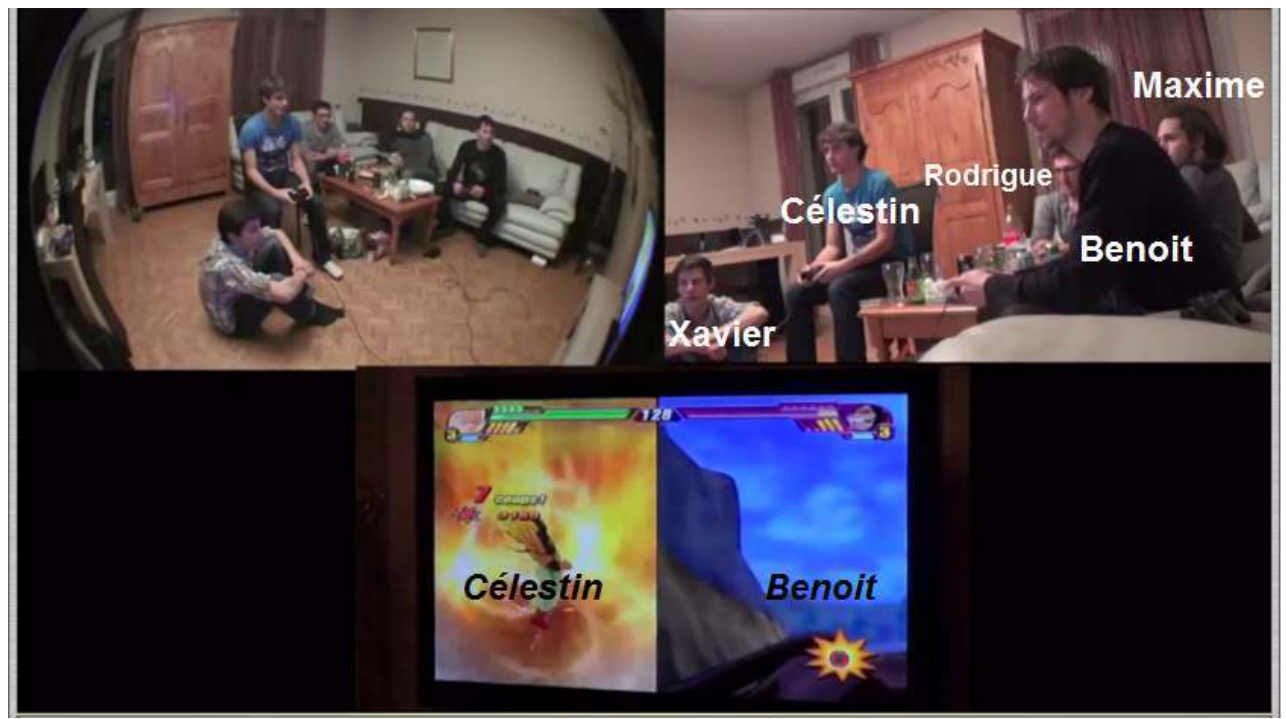

Image 4 : disposition des joueurs et spectateurs dans le jeu Dragon Ball

Pour cet extrait 2, les métadonnées que nous pouvons introduire au public-lecteur sont donc leurs prénoms: Célestin qui joue contre Benoit en mode «Duel», et Maxime, Rodrigue et Xavier qui sont spectateurs du jeu. L'objectif du jeu est de donner le plus de coups gagnants pour cumuler des points et gagner la partie. 


\section{2. Description précise de l'extrait choisi en lien avec la problématique étudiée}

17 Dans un second temps, avant de procéder à l'analyse linguistique et interactionnelle des extraits, nous complétons les métadonnées précédemment introduites par des descriptions plus précises des extraits sélectionnés, en cherchant à relier avec la problématique étudiée dans la communication ou l'article. Ces éléments de contexte supplémentaires permettent au public-lecteur de resituer l'extrait dans une temporalité de jeu plus large et à laquelle il n'a pas accès. Ils concernent aussi bien les actions en cours des joueurs et de leurs avatars, les règles et des spécificités du jeu dans l'extrait, l'énonciateur du tour d'encouragement ou d'instruction en relation avec les événements de l'extrait, ou encore un rappel du rôle de chacun dans l'extrait analysé.

\section{2. 1. Extrait $1:$ « vite vite vite/ vite vite vite/»}

18 Pour l'extrait 1 dans le jeu Mario, nous précisons au public-lecteur les éléments de contexte suivant :

- Du point de vue des actions : les joueurs rencontrent des difficultés à éviter différents obstacles. L'avatar de Léa vient juste de perdre une vie quand elle est tombée dans la lave. L'avatar de Dominique essaye d'avancer dans le jeu et ses co-équipiers lui laissent la place pour le faire. Les avatars de Lucas et Véro sont en position de bulle et flottent dans le paysage du jeu.

- Du point de vue des règles et spécificités du jeu : la position « en bulle » permet aux avatars de ne pas perdre une vie en étant touché par un bloc de pierre piquant. S'ils sont touchés par un des blocs ou par un autre avatar, ils sortent de la bulle et peuvent potentiellement tomber dans la lave, ou être touché par un bloc de pierre et perdre une vie.

- Du point de vue de l'énonciateur du tour : le risque d'être touché par un bloc de pierre est important à cet instant de la partie, et ce risque est à l'origine d'une séquence d'encouragement de la part de Lucas.

- Du point de vue des rôles de chacun (joueur vs spectateur) : Lucas et Véro sont en partie spectateurs, en position bulle ; Dominique est joueur et avance dans le jeu ; l'avatar de Léa vient de perdre une vie, il n'est pas encore revenu dans le jeu et ne revient qu'à la fin de l'extrait, Léa est donc principalement spectatrice.

\section{2. 2. Extrait $2:$ : allez :/ vas-Y benoit » ; « fais carré ENfoncé/ fais carré ENfoncé »}

19 De même, pour l'extrait 2 dans Dragon Ball, nous précisons au public-lecteur les éléments de contexte suivant :

20 - Du point de vue des règles et spécificité du jeu : Benoît a choisi comme avatar le Gorille géant, Célestin est incarné par Super Gogeta (cf. image 5). Cette donnée est importante pour l'analyse ensuite. 


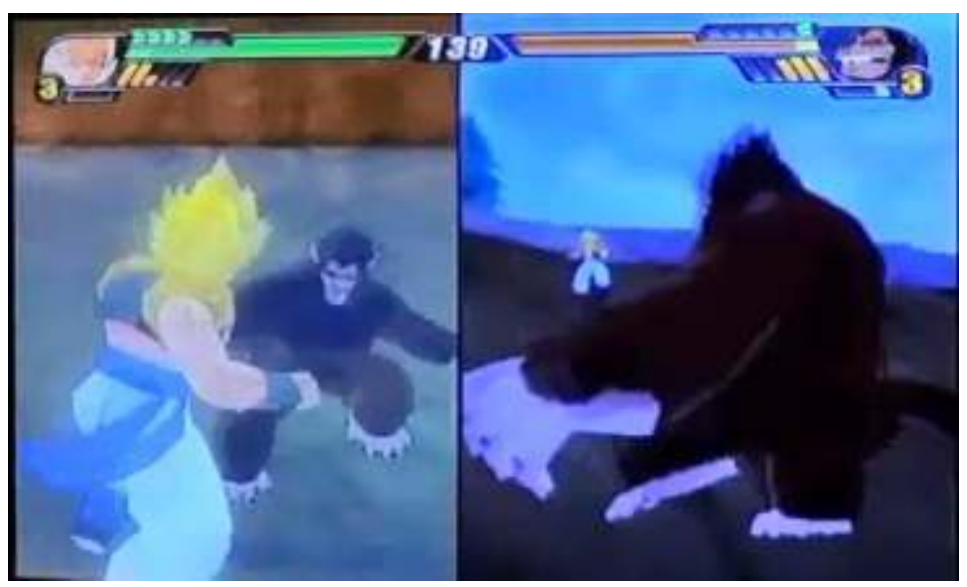

Image 5 : les deux avatars choisis par les joueurs (à gauche Super Gogeta, à droite le gorille géant)

- Du point de vue de l'énonciateur des tours : les deux énoncés sont prononcés par deux spectateurs. L'encouragement est produit par Rodrigue et l'instruction par Xavier. Ces deux tours sont adressés à Benoit.

- Du point de vue du rôle de chacun (joueur vs spectateur) : Rodrigue, Xavier et Maxime sont spectateurs ; Benoit et Célestin sont les deux joueurs.

À la différence de l'extrait 1, il n'est pas nécessaire ici de contextualiser les actions pertinentes dans le jeu, car le mode de jeu est celui du combat un à un et l'objectif du jeu décrit plus haut suffit à apporter les éléments d'informations au public-lecteur avant l'analyse de l'extrait.

De manière générale, les métadonnées et les descriptions précises des extraits permettent ainsi de guider le public-lecteur sur les éléments pertinents à regarder dans l'extrait vidéo joué pendant une communication orale et/ou à comprendre dans la transcription de l'extrait par la suite. Ce travail de contextualisation des extraits est effectué en amont par le chercheur. Il est donc important d'avoir une bonne connaissance du corpus enregistré, d'avoir conservé les informations sur les noms précis des jeux joués pendant les sessions (leur version en fonction de la console et de l'année d'enregistrement). Ensuite, nous mobilisons à la fois notre propre expérience et connaissance du jeu : c'est le cas dans Mario avec les explications données sur la position en bulle des avatars et les conséquences que cela peut avoir dans les actions du jeu; mais nous utilisons aussi les règles des jeux trouvées en ligne pour reconstituer des informations dont nous avons aucune connaissance préalable: c'est le cas dans Dragon Ball avec les noms des avatars choisis par les joueurs. Leur sélection sur l'écran en début de partie ne nous a pas permis d'obtenir les noms précis des avatars, il a fallu les rechercher sur des pages web en ligne qui répertorient la liste des avatars disponibles dans cette version du jeu de Dragon Ball sur cette console.

Après avoir donné ces éléments de contextualisation, nous jouons les extraits vidéo (lors d'une communication orale uniquement) et nous enchaînons sur la transcription des extraits et leurs analyses. Là encore, il est indispensable de prendre en compte certaines ressources multimodales disponibles dans l'interaction entre les joueurs, ce que nous allons approfondir dans la section suivante. 


\section{Prendre en compte les ressources multimodales}

[The Contextualization of Language]. "This volume suggests a novel treatment of context in the analysis of everyday interaction. On a theoretical level, it advocates a switch of focus from 'context' as a preestablished, monolithic category which constringes coparticipants' verbal and nonverbal behaviour, to an active notion of 'contextualization': in order to make oneself understood, participants have to establish and maintain those shared contextual frames which in turn are relevant to the local interpretation of their verbal and nonverbal activities. On an empirical level, the volume contains exemplary analyses that show how participants employ 'contextualization cues' of prosodic (rhythm, intonation, tempo, etc.) or nonverbal (gaze, gesture, etc.) nature in order to 'achieve context " (Auer \& Di Luzio, 1992).

La prise en compte des ressources multimodales est mise en évidence dans les transcriptions verbales et non verbales que nous réalisons à partir des extraits vidéo. En Analyse Conversationnelle, la transcription des données permet de rendre compte de l'organisation séquentielle et interactionnelle des séquences choisies, et de construire ensuite notre analyse. Nous n'aborderons pas ici la méthodologie de transcription des phénomènes vocaux et verbaux ; nous préférons nous focaliser sur les contraintes liées à la transcription multimodale des données, c'est-à-dire à la transcription des phénomènes non verbaux (action non verbale, gestes, mimiques, etc.). En effet, nous observons trois contraintes fortes lorsque nous sommes confrontés à un travail de transcription multimodale :

- $\mathbf{1}^{\text {ère }}$ contrainte : il est pertinent de transcrire la multimodalité pour assurer une compréhension de l'analyse la plus claire, la plus compète mais la plus brève possible aussi sans jouer la vidéo (pour une diffusion dans un article imprimé par exemple).

- $2^{\text {ème }}$ contrainte $:$ il est difficile de généraliser les formats de transcriptions multimodales en Analyse Conversationnelle. Il n'y a pas de convention de transcription multimodale unique dans le domaine, elle peut dépendre des données analysées.

- $3^{\text {ème }}$ contrainte : la transcription multimodale de certains éléments est parfois non pertinente. Il ne faut donc pas tout transcrire. Nous devons faire des choix en fonction de la problématique de recherche et rendre compte des ressources multimodales rendues pertinentes du point de vue des joueurs, et à partir desquelles nous allons construire notre analyse.

Dans cette section, nous allons donc problématiser la prise en compte de ressources multimodales, d'une part dans la transcription, et d'autre part, pour l'analyse. Nous reprendrons les extraits 1 et 2 introduits dans la partie 3 précédente.

\section{1. Dans la transcription (forme)}

"The inherent turn-by-turn contextuality of conversation is a vital resource for the construction of understanding in interaction. Since each action will be understood as responsive to the previous one, the understanding that it displays is open for inspection " (Heritage, 2010: 210-211).

Les ressources multimodales dans la transcription de nos extraits de jeux vidéo sont établies à partir de la convention de Lorenza Mondada $(2018)^{3}$ que nous avons adaptée et que nous n'utilisons pas dans sa totalité. Ainsi, Adom, Aluc, Aben et Acel correspondent à l'avatar du joueur associé (Dominique, Lucas, Benoit ou Célestin). Aussi un symbole ( \% ou 
$\S$ ou \$) est associé à chaque joueur (ou spectateur) afin d'aligner temporellement le début et la fin d'une action d'un joueur ou de son avatar.

Les événements observables à l'écran (indépendamment d'un joueur ou d'un avatar) sont alignés temporellement par le symbole \# associé à l'abréviation « ecr ». Dans la situation de jeu sur Dragon Ball (extrait 2), l'écran est divisé en deux, un pour chaque joueur. Dans ce cas-là, nous spécifions le joueur à l'écran concerné « ecrC " par exemple pour « écran de Célestin ». Enfin, nous décrivons textuellement les actions et informations pertinentes. D'un point de vue méthodologique, ce travail de description des actions précisément aligné à la transcription est réalisé à l'aide du logiciel ELAN ${ }^{4}$ qui nous permet d'annoter rigoureusement les événements multimodaux au signal vidéo. Nous effectuons ensuite une conversion au format texte pour l'obtenir dans une mise en page adaptée à une présentation orale ou un article.

Ainsi, nous obtenons les transcriptions multimodales suivantes (lignes grisées) pour les extraits 1 et 2 .

\section{1. 1. Extrait $1:$ « vite vite vite/ vite vite vite/»}

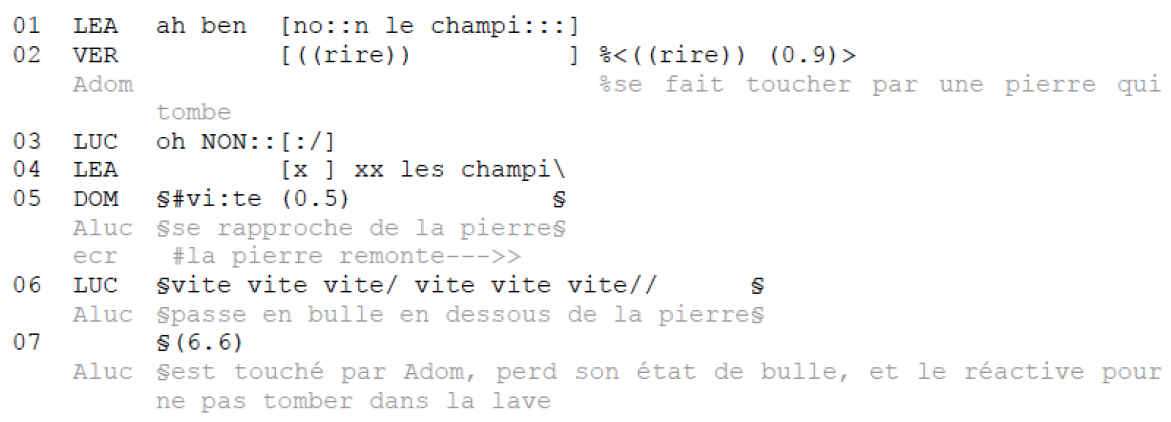

Extrait 2 " « allez:/vas-Y benoit »; "fais carré ENfoncé/ fais carré ENfoncé »

$01 \mathrm{XxX} \#<($ inaud.) (2.3) $>$

ecrC \#(( 8 coups, 8640 points $)$

$02 \quad(1.3)$

03 BEN \$+je déteste ce gorille

benG \$rronce les sourcils

Aben tse plie sous les coups de l'adversaire

$04+(0.6)$

Aben +saute sur place et boxe dans le vide-->

05 ROD \#allez:/ vas-y benoit

ecrc \#le nombre de coups portés monte rapidement de 1 à 5

06 XAV [fais carré Enfoncé] fais carré ENfoncé

07 BEN [+\#XXXx ]

benG +\#mouvement du buste en arrière

ecrc \#((6 coups, 3170 points $))$

$08 \div(0.2)$

Acel sattaque $1^{\prime}$ avatar de Ben

09 BEN moi/

31 Cependant, nous nous posons souvent la question quant à l'objectivité et la clarté des descriptions textuelles que nous réalisons dans ces transcriptions multimodales. D'un point de vue méthodologique, nous choisissons donc d'utiliser très régulièrement des captures d'écran des actions décrites afin de rendre compte, le plus fidèlement possible au public-lecteur, des types des ressources sur lesquelles nous basons notre analyse. Après, dans le cadre d'un article imprimé, nous avons le choix de faire apparaitre ces captures soit alignées dans les transcriptions (cf. image 6 ci-dessous), soit au fur et à mesure des paragraphes d'analyse par la suite. Dans le cadre d'une communication orale, notre décision est plus simple car nous avons la possibilité de faire apparaitre les captures 
écran à côté des transcriptions au moment où nous déroulons l'analyse linguistique et interactionnelle à l'oral.

Image 20000161000040CD0000538D2B3D0093.wmf

Image 6 : transcription avec les captures d'écran alignées des actions décrites

Avec ce type de corpus d'interactions en situation de jeux vidéo, nous faisons généralement le choix dans nos articles d'insérer les captures d'écran au fur et à mesure des paragraphes de l'analyse car d'une part, nous pensons que ce choix allège la longueur de la transcription et facilite sa lisibilité au lecteur de l'article, et d'autre part, nous réintroduisons ainsi des éléments de contexte complémentaires dans une forme, cette fois-ci, iconographique à l'intérieur des analyses, comme nous le verrons dans la section suivante qui concerne justement la prise en compte des ressources multimodales pour l'analyse interactionnelle.

\section{2. Pour l'analyse interactionnelle (fond)}

La notation des ressources multimodales au niveau de la transcription de la conversation est nécessaire pour rendre compte de la séquentialité et de la temporalité des actions, regards, gestes, postures, etc. de l'activité analysée dans son ensemble. C'est à partir de la transcription que nous construisons ensuite notre analyse linguistique et interactionnelle. Les numéros de lignes qui précèdent chaque tour de parole dans la transcription nous permettent de faire référence aux productions orales des locuteurs dans l'analyse mais de faire aussi référence aux descriptions multimodales (lignes grisées).

34 Nous proposons ainsi les analyses des extraits 1 et 2 . Ces analyses s'inscrivent dans la perspective de notre recherche sur les encouragements et les instructions dans les interactions de jeux vidéo. Nous montrerons de quelle manière nous mobilisons les ressources multimodales décrites dans la transcription mais aussi l'importance de la contextualisation des extraits présentés en amont de l'analyse.

\section{2. 1. Extrait $1:$ « vite vite vite/ vite vite vite/»}

Léa exprime sa frustration contre «le champi » (ligne 1) d'avoir perdu une vie en tombant dans la lave, après avoir été touchée par l'avatar de Lucas qui l'a empêché par inadvertance de sauter par-dessus la lave. Véro rie (ligne 2) et partage ainsi le caractère amusant de la situation. Au même moment, l'avatar de Dom est touché par une pierre qui tombe (ligne 2, cf. image 7). Il perd seulement une option que son avatar avait obtenu (i.e. la taille plus grande) et redevient « petit Mario » comme au début d'une partie. 


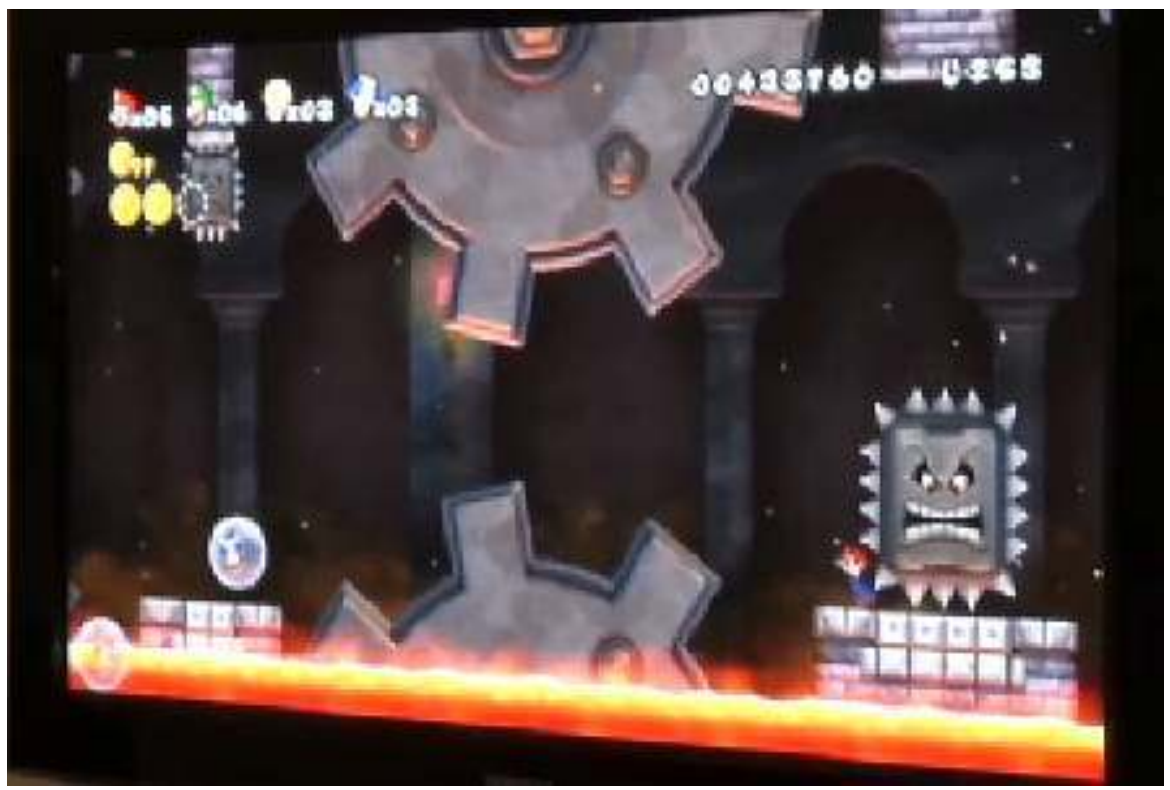

Image 7 : l'avatar de Dom est touché par un bloc de pierre

évalue négativement l'événement survenu sur l'avatar de Dom (ligne 3). Leur chance de gagner en équipe diminue en conséquence. En effet, les avatars de Véro et Lucas sont en bulle, et celui de Léa vient juste de perdre une vie et n'est pas encore revenu dans le jeu. Si le dernier avatar toujours dans le jeu perd aussi une vie (i.e. l'avatar de Dom), alors le jeu s'arrête et recommence au début de ce monde.

Ensuite, la pierre remonte lentement au plafond (ligne 5, cf. image 8) : les joueurs doivent profiter de ce moment pour avancer dans le jeu et passer cet obstacle. Il s'agit d'un moment crucial dans la partie (tension, pression de réussir). Dominique produit un premier encouragement adressé à son avatar (ligne 5). Il se positionne à cet instant comme spectateur des actions de son avatar et l'avatar comme participant qui peut réagir/répondre et endosser la responsabilité (para-interaction).

En même temps, l'avatar de Lucas se rapproche de la pierre en position bulle, il doit donc rester prudent pour ne pas se faire toucher par la pierre ou par l'avatar de Dominique.

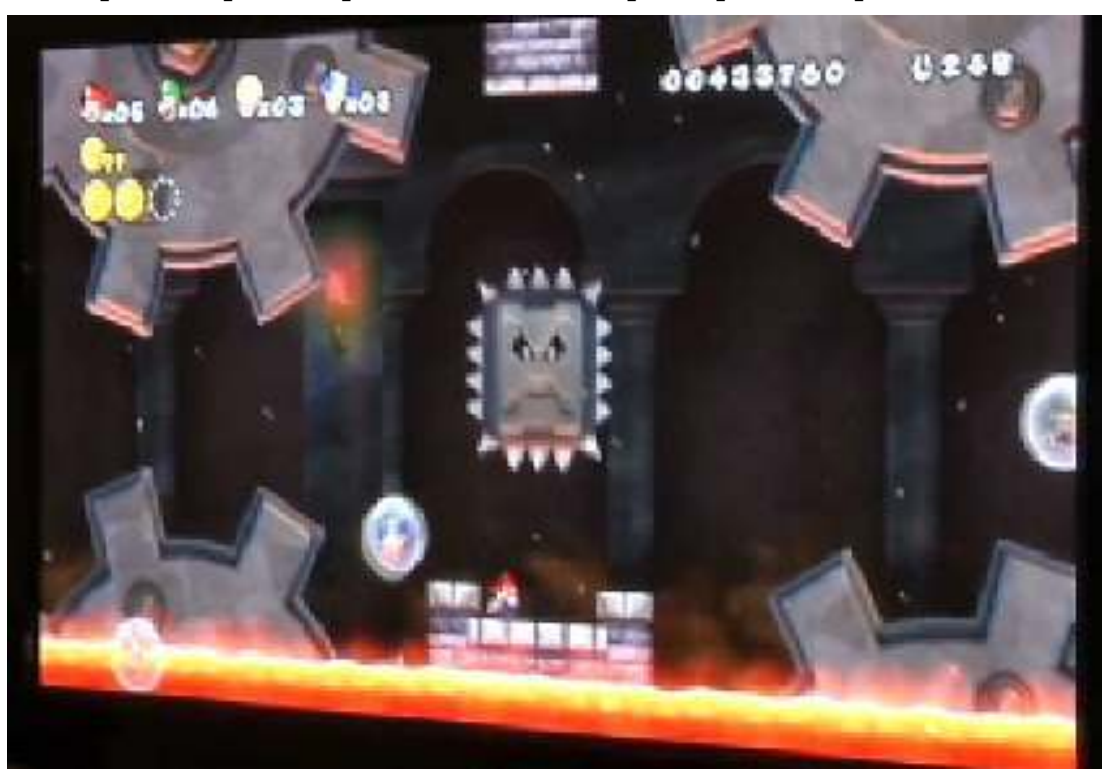

Image 8 : la pierre remonte, l'avatar de Lucas se rapproche 
Ligne 6, Lucas enchaine le tour suivant, en utilisant le même adverbe que Dominique, répété six fois " vite vite vite/ vite vite vite/ ». Il passe simultanément sous la pierre (cf. image 9). Nous analysons le tour de Lucas comme à la fois adressé : a) à l'avatar de Dominique afin qu'il puisse avancer rapidement devant lui et éviter ainsi de le toucher (ce qui arrive juste après, pendant la longue pause de 6.6 secondes ligne 7 , cf. image 10), et également que la pierre ne retombe pas sur son avatar; b) à son propre avatar pour l'encourager à passer l'obstacle avec succès.

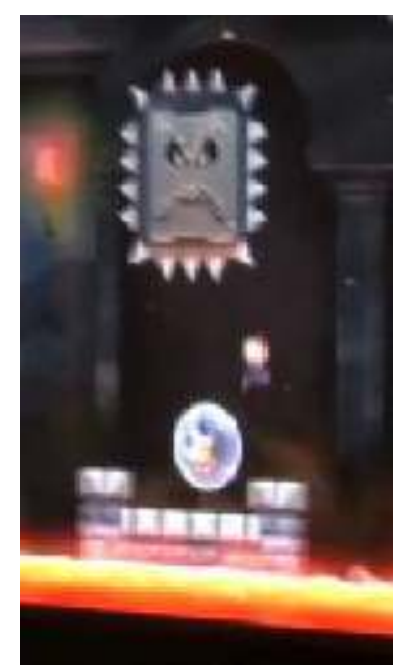

Image 9 : L'avatar de Lucas passe sous la pierre

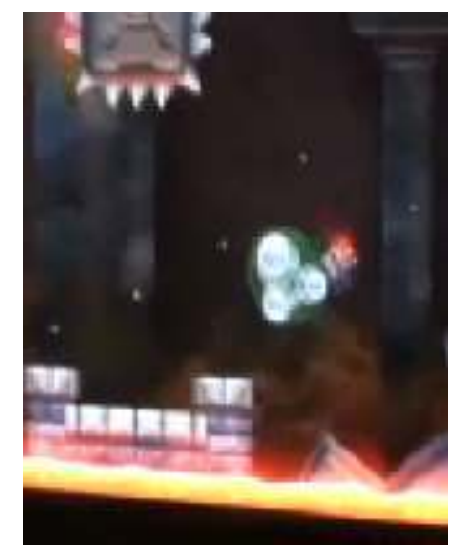

Image 10 : Les avatars de Lucas et Dominique se touchent

Pour résumer, l'analyse du tour d'encouragement « vite vite vite/ vite vite vite » produit par Lucas est liée

- à ce que les avatars ne se fassent pas toucher de manière générale dans le jeu ;

- à ce que l'avatar de Dominique ne soit pas touché sur la fin de l'extrait car il est en petite taille ;

- à éviter par conséquent de recommencer ce monde au début ;

- à la position bulle de certains avatars dans le jeu

- à l'avatar de Léa qui vient de perdre une vie et n'est pas encore de retour dans le jeu

41 Ainsi, au-delà de la description syntaxique et prosodique réalisée sur ce tour d'encouragement, nous analysons aussi son contexte et son environnement séquentiel dans lequel il est produit (pour en dégager par exemple des formats interactionnels 
similaires, récurrents, comparables ou non). Pour cela, nous décrivons les ressources multimodales pertinentes, les différentes actions et spécificités du jeu.

\section{2. 2. Extrait 2 : « allez :/ vas-Y benoit » ; « fais carré ENfoncé/ fais carré ENfoncé »}

Les spectateurs interviennent de façon inaudible et en chevauchement (ligne 1), pendant que l'avatar de Célestin attaque celui de Benoit et accumule des points. L'écran de Célestin indique alors 8 coups (donnés) et 8640 points acquis (cf. image 11); alors que Benoit voit son énergie se vider rapidement (cf. image 12).

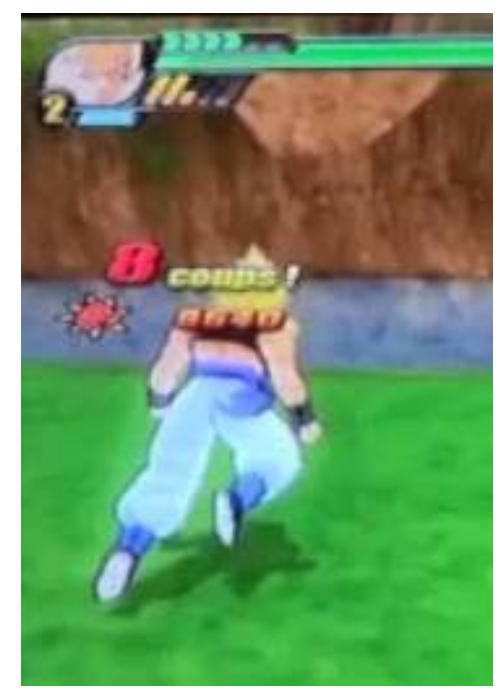

Image 11 : 8 coups (donnés) et 8640 points acquis sur l'écran de Célestin
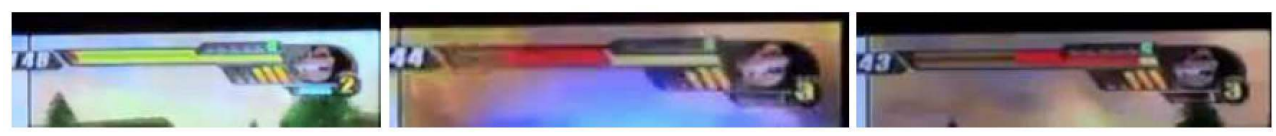

Image 12 : Benoit perd de l'énergie très rapidement

Benoit répond verbalement à ce déséquilibre et se plaint de son avatar «je déteste ce gorille » (ligne 3). On observe du point de vue non verbal qu'il fronce en même temps les sourcils. Par ce tour, il rejette ainsi la responsabilité du mauvais score de son avatar. Ce dernier est justement en train de se plier sous les coups de l'adversaire. Ensuite, l'avatar de Benoit boxe dans le vide sans atteindre son opposant (ligne 4). Les actions dans le jeu et les commentaires de Benoit montrent localement un affaiblissement de son engagement dans le jeu.

5 et 6 , deux spectateurs interviennent :

a) Rodrigue avec un encouragement « allez :/ vas-y benoit » pour remotiver Benoit,

b) Xavier avec une instruction technique : "fais carré enfoncé/ fais carré enfoncé » pour aider Benoit à mieux attaquer l'adversaire, ce qu'il n'a pas réussi à faire encore.

Nous observons une asymétrie dans les connaissances de ce jeu. En effet, Xavier se positionne ici comme quelqu'un qui sait et Benoit comme quelqu'un qui ne sait pas (et qui a donc besoin d'être instruit et encourager).

Parallèlement, l'écran de Célestin indique encore une augmentation rapide du nombre de coups portés sur son adversaire (ligne 5). Benoit a alors un mouvement du buste en arrière et Célestin gagne 3170 points après 6 coups donnés à la suite (ligne 7 , cf. image 13). 


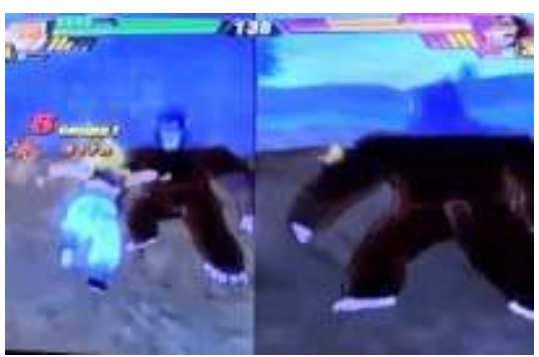

Image 13 : l'écran de Célestin indique 3170 points et 6 coups donnés

\section{carré enfoncé/ fais carré enfoncé » est donc liée}

- aux nombreux coups reçus par l'avatar de Benoit

- à la perte d'énergie rapide de l'avatar de Benoit dans le jeu

- à la plainte de Benoit sur son avatar indique assez précisément une action (manipulation technique, une stratégie à adopter) que le joueur doit faire maintenant et qu'il n'a visiblement pas ou pas suffisamment fait jusqu'à ce moment du jeu.

Selon la temporalité du jeu, l'instruction est dans ce cas très concise, directive et très bien positionnée. Le joueur pour sa part peut ou non suivre l'instruction. L'action instruite est généralement accomplie sans aucun commentaire. Le joueur ne peut pas non plus toujours répondre à l'instruction, verbalement ou dans d'autres modes interactifs, sans que l'absence de réponse soit marquée dans l'interaction.

En revanche, Rodrigue n'instruit pas une action, mais il fait référence à la façon de jouer de Benoit. Il l'incite à « continuer » et tente donc de le remotiver. La directive utilisée dans ce tour est très vague et n'indique pas une action spécifique (« vas-y »). Il ne montre pas une expertise particulière ou une asymétrie dans la connaissance du jeu.

est plutôt utilisé pour soutenir le joueur, pour montrer lengagement de Rodrigue dans le jeu malgré sa position de spectateur et donc pour rassembler les participants en tant que membre d'un groupe (joueurs et spectateurs). Schilling (2001:309) décrit comme «encouragement " ('Anspornen') «toutes les activités de l'entraîneur qui tendent à convaincre le joueur que la tâche à venir est généralement résolvable ».

55 C'est ce que Rodrigue fait ici dans une forme plutôt minimale. Benoit ne répond pas directement, ni verbalement, ni dans d'autres modes interactionnels ou dans le jeu. Mais il ne démissionne pas, il continue à jouer et progresse dans le jeu. Donc, si la réponse est 
de montrer « l'effort », comme le suggère Reynolds, l'effort est visible au fil du temps du jeu.

\section{Conclusion}

A partir de nos données d'interactions entre en situation de jeux vidéo multi-joueurs, nous avons cherché à problématiser la question de la contextualisation des extraits et de la prise en compte des ressources multimodales pour une analyse des pratiques interactionnelles entre joueurs. Notre étude a permis de mieux comprendre les manières dont tout ce travail de contextualisation transforme à la fois le travail de recherche, le travail de recueil du corpus, mais aussi le traitement analytique des données et la présentation des extraits lors de communication pendant des conférences ou lors de la rédaction d'article pour des publications scientifiques.

Dans ces corpus de jeux vidéo, le travail de contextualisation nous semble encore plus difficile, complexe que pour d'autres corpus vidéo (d'interactions privées ou professionnelles) étudiés dans nos recherches. En effet, nous devons bien sûr nous adapter au public, au lecteur (dans les articles), tout en étant très contrainte par le temps de présentation (souvent 20 min à l'oral). De notre point de vue, il nous parait insuffisant de ne présenter qu'un seul extrait pour rendre compte du phénomène analysé (par exemple celui des encouragements, instructions). Aussi, malgré la complexité du dispositif (au minimum 1 vue écran et jusqu'à 4 vues écran selon le contexte de jeu et le nombre de joueurs en simultané), nous avons remarqué que le travail de contextualisation n'est pas plus difficile à mener dans les situations à plusieurs écrans de joueurs. Par exemple, dans l'extrait 1, nous avons analysé les actions sur un seul écran commun aux 4 joueurs mais il s'est avéré que les informations précisées sur les métadonnées générales du jeu ainsi que les explications des spécificités de l'extrait analysé ont rendu le travail de contextualisation assez long et assez riche à réaliser en amont. Pour rappel, sur l'analyse des séquences d'encouragement, ou d'instruction, il nous semble indispensable de contextualiser les informations suivantes qui donneraient du sens à la présentation de l'extrait et/ou à son analyse : le type de jeu joué, ses règles, ses options spécifiques, les relations entre les joueurs, les rôles de chacun à l'instant de l'extrait, et aussi les actions en cours dans l'univers du jeu lui-même (un objet qui tombe, un avatar touché, les scores à l'écran d'un des joueurs, etc.)

Par ailleurs, nous prenons en compte les retours éventuels du public-lecteur suite à chaque présentation ou évaluation (d'articles). Nous avons eu souvent par exemple des remarques sur le passage trop rapide sur des extraits, sur une compréhension du jeu difficile même avec des explications et les éléments contextuels, ou encore des relecteurs (sur nos articles) qui suggéraient d'ajouter des indications graphiques pour aider le lecteur à se repérer sur les captures d'écran insérées dans les analyses, dans les transcriptions (ex: flèches, cercles sur l'élément focus). Aussi, le format "publication écrite" permet d'avoir un espace plus grand pour rédiger ces éléments de contextualisation avant et pendant les analyses.

Egalement, nous souhaitons souligner l'importance de la langue de présentation de nos recherches sur les jeux vidéo. En effet, nous sommes amenée à présenter ou publier nos travaux en français, anglais ou allemand et nous avons donc dû mener des recherches précises sur le vocabulaire propre à chaque jeu, à chaque règle de jeu vidéo dans la langue 
de publication pour rester fidèles aux spécificités des jeux analysés lors de leur contextualisation.

Enfin, nous nous interrogeons sur la part accordée (par le public-lecteur) ou à accorder (par le chercheur) à ces éléments de contexte par rapport à l'analyse linguistique et interactionnelle que nous réalisons. Réellement, que fait le public-lecteur de ces éléments de contexte à l'instant $t$ de la présentation ou à la lecture de l'article? Aussi, immergé pleinement dans son corpus de jeux vidéo, jusqu'à quel point le chercheur a-t-il conscience de la complexité du corpus qu'il présente? Deux questions qui restent toujours à notre esprit lorsque nous présentons des analyses sur nos données de jeux vidéo et sur lesquelles il est nécessaire d'être toujours vigilante.

\section{BIBLIOGRAPHIE}

Auer, Peter et Di Luzio, Aldo (Eds.), (1992), "The contextualization of language", Amsterdam /

Philadelphia, John Benjamins Publishing.

Baldauf-Quilliatre, Heike et Colón de Carvajal, Isabel (2015). Is the avatar considered as a

participant by the players? A conversational analysis of multi-player videogames interactions. In: PsychNology Journal, 13(2-3), p. 127-147.

Baldauf-Quilliatre, Heike et Colón de Carvajal, Isabel (accepté). « The spectator as coach: instructing, encouraging and giving advices » In: Social Interaction. Video-Based Studies of Human Sociality.

Baldauf-Quilliatre, Heike et Colón de Carvajal, Isabel (en préparation). « Jouer aux jeux vidéo comme activité sociale : Le rôle du spectateur. In : Langage et Société

Colón De Carvajal, Isabel (2013). Choix méthodologiques pour une analyse de conversation en situation de jeu vidéo. COLDOC 2012, Traitement de corpus : outils et méthodes. Actes du colloque linguistique de doctorants et jeunes chercheurs du laboratoire Modyco, p. 20-35.

Colón de Carvajal, Isabel (2016). The body as a controller to move its videogame avatar: players practice between knowledge, learning and resistance to the human-machine interaction device. Conference RIAL 2016 - L'être de langage, entre corps et technique : nouvelles données, nouvelle donne? Université Paul Valery Montpellier 3.

Groupe ICOR (2006). La démarche ethnographique. Available at CORINTE : [icar.univ-lyon2.fr/ projets/corinte/recueil/demarche_ethnographique.htm] [accessed October, 2017]

Heritage, John (2010). "Conversation Analysis: Practices and methods", In Qualitative Sociology (3rd edition) (David Silverman, ed.), London, Sage, p. 208-230.

Mondada, L. (2011). The situated organization of directives in French: Imperatives and action coordination in video games. Nottingham French Studies, 50(2), p. 19-50.

Mondada, Lorenza (2012). Coordinating action and talk-in-interaction in and out of video games. The appropriation of media in everyday life, p. 231-270. 
Mondada, Lorenza (2014). Instructions in the operating room: How surgeons direct their assistant's hands. In: Discourse Studies 16(2), p. 131-161.

Mondada, Lorenza (2018). Multiple Temporalities of Language and Body in Interaction:

Challenges for Transcribing Multimodality, Research on Language and Social Interaction, 51(1),

p. 85-106

Piirainen-Marsh, Arja et Tainio, Liisa (2009a): Collaborative game-play as a site for participation and situated learning of a Second Language. Scandinavian Journal of Educational Research, 53(2),

p. $167-183$.

Piirainen-Marsh, Arja et Tainio, Liisa (2009b): Other- repetition as a resource for participation in the activity of playing a video game. The Modern Language Journal, 93(2), p. 153- 169.

Schilling, Marcel (2001). Reden und Spielen. Die Kommunikation zwischen Trainern und Spielern im gehobenen Amateurfußball. Tübingen: Narr.

Tekin, Burak S. et Reeves, Stuart (2017, May). Ways of spectating: unravelling spectator participation in Kinect play. In: Proceedings of the 2017 CHI Conference on Human Factors in Computing Systems, p. 1558-1570.

\section{ANNEXES}

\section{Convention de transcription des phénomènes vocaux-verbaux}

[ ] parole en chevauchement

八 intonation montante ou descendante

${ }^{\circ}$ voix murmurée, plus basse

: : : allongement du son ou de la syllabe

p`tit élision

trouv- troncation

xxx syllabe incompréhensible

$=$ enchainement immédiat

() transcription incertaine

(()) commentaires

\& tour du même locuteur intercalé par un chevauchement

(.) micro-pause

(0.6) pause quantifiée

\section{NOTES}

1. Page Web de l'équipe InSitu au laboratoire ICAR : http://icar.univ-lyon2.fr/pages/equipe1.htm

2. Page web du projet Ludespace : http://citeres.univ-tours.fr/spip.php?article1267 
3. Pour retrouver la convention de transcription multimodale de L. Mondada (2008) en détail : http://icar.univ-lyon2.fr/projets/corinte/documents/

convention_transcription_multimodale.pdf

4. The Language Archive, Max Planck Institute for Psycholinguistics : https://tla.mpi.nl/tools/ tla-tools/elan/

\section{RÉSUMÉS}

Notre travail s'inscrit dans les ancrages théoriques de l'ethnométhodologie et de l'analyse conversationnelle. Notre intérêt est avant tout d'analyser les pratiques interactionnelles entre joueurs en situation de jeux vidéo (Colón de Carvajal, 2013, 2016; Mondada, 2011, 2012 ; Piirainen-Marsh \& Tainio, 2009a et b) ; et d'autre part, de comparer différents jeux sur le plan de leur organisation interactionnelle (les cadres de participation) ainsi que leur structure séquentielle et multimodale (l'organisation de l'espace et la gestion des objets). Á partir des pratiques de joueurs, nous questionnons les problèmes et contraintes rencontrés, d'une part lors des analyses de ces données en situation de jeux vidéo, et d'autre part lors de présentations académiques de nos recherches. Une étude en cours, sur les séquences d'encouragement et d'instruction dans les interactions entre joueurs, nous permet de montrer qu'il est indispensable de contextualiser le type de jeu joué, ses règles, ses options spécifiques parfois, les relations entre les joueurs, les rôles de chacun à l'instant de l'extrait, et aussi les actions en cours dans l'univers du jeu lui-même (un objet qui tombe, un avatar touché, etc.) qui donneraient du sens à l'analyse. Ces contextualisations et prises en compte des ressources multimodales permettent d'expliquer et de décrire plus clairement et objectivement le phénomène linguistique, l'action ou encore la problématique analysée.

Our research is embedded in the theoretical issues of ethnomethodology and conversational analysis. Our interest is above all to analyze the interactional practices between players in a video game situation (Colón de Carvajal, 2013, 2016, Mondada, 2011, 2012, Piirainen-Marsh \& Tainio, 2009a and b); and on the other hand, to compare different games in terms of their interactional organization (participation frameworks) as well as their sequential and multimodal structure (the organization of space and the management of objects). From the practices of players, we question the problems and constraints encountered, on the one hand during the analysis of these data in a video game situation, and on the other hand during academic presentations of our research. A work in progress on encouragement's and instruction's sequences in interactions between players, allows to show that it is essential to contextualize the type of game played, its rules, its specific options, the relationships between players, the roles of each player at the moment of the extract, and also the current actions in the universe of the game itself (a falling object, a touched avatar, etc.) that would give meaning to the analysis. These contextualizations and these considerations of multimodal resources make it possible to explain and describe more clearly and objectively the linguistic phenomenon, the action or the problem analyzed. 
INDEX

Keywords : interaction, videogames, pratices, multimodal resources, constraint for analysis, constraint for academic presentation

Mots-clés : interaction, jeux vidéo, pratiques, ressources multimodales, contrainte pour l'analyse contrainte pour une présentation académique

\section{AUTEUR}

\section{ISABEL COLÓN DE CARVAJAL}

École Normale Supérieure de Lyon Laboratoire ICAR (UMR 5191) 